\title{
Simultaneous Measurement of Changes in Thickness and Refractive Index of Weakly Absorbing Self-Standing Solid Films Using Optical Interferometry
}

\author{
Nalini Easwar \\ Smith College, neaswar@smith.edu \\ R. Fantini \\ Raytheon Company \\ E. Willis \\ Teledyne Brown Engineering
}

Follow this and additional works at: https://scholarworks.smith.edu/phy_facpubs

Part of the Physics Commons

\section{Recommended Citation}

Easwar, Nalini; Fantini, R.; and Willis, E., "Simultaneous Measurement of Changes in Thickness and Refractive Index of Weakly Absorbing Self-Standing Solid Films Using Optical Interferometry" (2001). Physics: Faculty Publications, Smith College, Northampton, MA.

https://scholarworks.smith.edu/phy_facpubs/43 


\section{Simultaneous measurement of changes in thickness and refractive index of weakly absorbing self-standing solid films using optical interferometry}

Cite as: Review of Scientific Instruments 72, 2842 (2001); https://doi.org/10.1063/1.1372676

Submitted: 01 August 2000 . Accepted: 23 March 2001. Published Online: 23 May 2001

N. Easwar, R. Fantini, and E. Willis

\section{ARTICLES YOU MAY BE INTERESTED IN}

Simultaneous measurement of refractive-index and thickness for optical materials by laser feedback interferometry

Review of Scientific Instruments 85, 08311 (2014); https://doi.org/10.1063/1.4892465

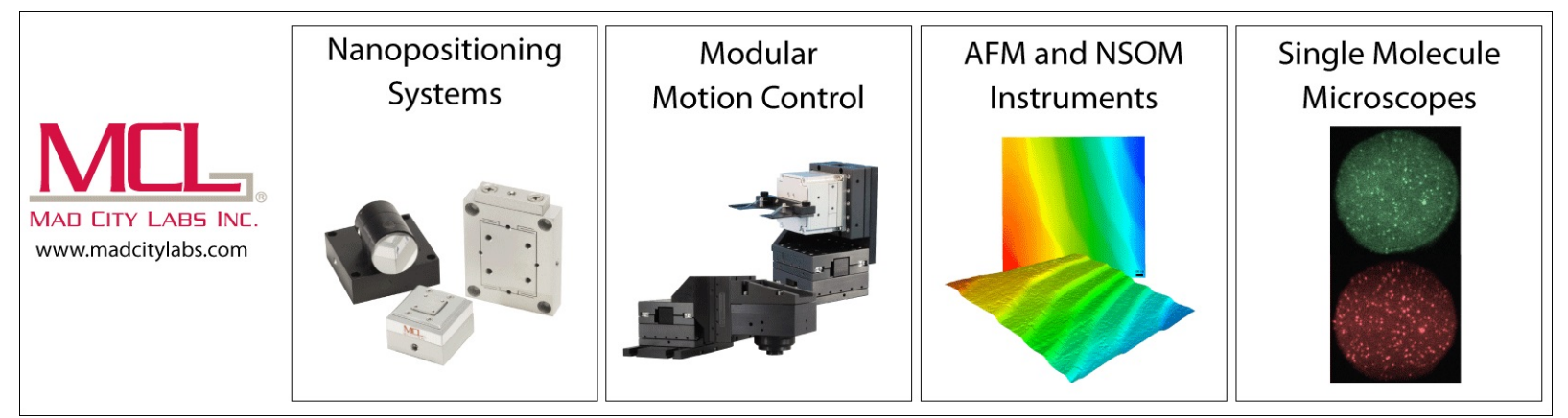




\title{
Simultaneous measurement of changes in thickness and refractive index of weakly absorbing self-standing solid films using optical interferometry
}

\author{
N. Easwar, ${ }^{\text {a) }}$ R. Fantini, ${ }^{\text {b) }}$ and E. Willis ${ }^{\mathrm{c})}$ \\ Department of Physics, Smith College, Northampton, Massachusetts 01063
}

(Received 1 August 2000; accepted for publication 23 March 2001)

\begin{abstract}
A nondestructive optical interferometric method, which enables a direct and simultaneous measurement of small changes in both thickness and refractive index of thin films, is described. Optical interferometric methods are sensitive to the changes in the optical path length (the product of the refractive index and the physical thickness) through the film. Thus, when the film is subjected to changing environmental conditions such as changing temperature or humidity, it is a challenge to determine the change in thickness and the change in refractive index separately, using interferometry. By simultaneously monitoring two different sets of interference fringes, i.e., transmission and reflection fringes, we have been able to successfully address this challenge. Our measurements on well-characterized pedigree glass samples are presented. The results agree well with the expected values for these samples, supporting the feasibility of this characterization technique to new materials. Self-standing, transparent (weakly absorbing) films ranging in thickness from tens to hundreds of microns can be characterized from direct measurements. (C) 2001 American Institute of Physics. [DOI: 10.1063/1.1372676]
\end{abstract}

The properties of thin films could differ greatly from those of bulk samples of the same material. Hence, measurements must be made on the material in thin-film form. The changes in thickness of thin films due to changing temperature, for example, are on the order of optical wavelengths and, hence, demand high precision in the measurements. Using an optical method allows for this kind of precision while being nondestructive to the film. The challenge that arises with an optical interferometric method comes from the fact that the critical parameter affecting the measurements is the optical thickness of the film, which is the product of the refractive index and the physical thickness.

Interferometric methods have been commonly used for thin-film characterization. ${ }^{1-5}$ It is important to note here that most of these techniques are used to measure the absolute thickness and/or refractive index (not changes in these parameters) and most often, the setup is not easily adaptable to subjecting the film to changing environmental parameters such as temperature or humidity. In general, the methods used to measure changes in either of these two characteristics, namely, thickness $(t)$ or refractive index $(n)$, usually end up measuring changes in the optical thickness $\Delta(n t)$ of the film. From this, the change in either thickness $(\Delta t)$ or refractive index $(\Delta n)$ is extracted by either (a) an independent measurement of the change in the other parameter, using a bulk sample of the material, ${ }^{6}$ or (b) by using an independent model for estimating or calculating the change in the other parameter. ${ }^{2}$ The common optical method for characterizing thin films is ellipsometry. ${ }^{7-9}$ In this method, the change

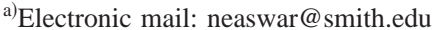

${ }^{b)}$ Currently at Raytheon Company, Tewksbury, MA 01876.

${ }^{c}$ Currently at Teledyne Brown Engineering, Huntsville, AL 35807.
}

in polarization of monochromatic light on reflection is measured at several wavelengths and angles of incidence. Ellipsometry can be used for films on substrates and works well for very thin films. However, the analysis of data for thicker films (relative to the wavelength used) becomes increasingly problematic. Some other methods used for thin-film characterization are as follows: (a) soft $\mathrm{x}$-ray reflectance measurements used for very thin films, ${ }^{10}$ (b) the measurement of the angular modulation of the reflected intensities from the front and back surfaces of the film, ${ }^{11}$ (c) the combined measurement of the reflectivity and the coefficient of reflectivity differentiated with respect to the incident angle of light, ${ }^{12}$ and (d) the measurement of transmittance spectra. ${ }^{13-17}$ In most of the methods above, the optical constants are obtained from a fitting procedure to appropriate theoretical calculations. An optical interferometric method using a combination of a modified Jamin interferometer and a thin-wedge technique has been reported for determining the thickness and optical index directly. ${ }^{18}$ The method described in this note is useful to make a simultaneous measurement of the changes in both the thickness and optical index characteristics of the films with changing temperature. Films from a few microns to several hundred microns in thickness can be studied by using a novel combination of two very fundamental sets of interference patterns from a film, i.e., reflection and transmission fringe patterns.

As discussed above, the overall change in the optical path through the film must be separated into changes attributed to each of the two parameters. Therefore, in designing this experiment it was necessary to incorporate two independent methods of measuring the changes in the film. A combination of transmission and reflection interferometry provides the solution. 


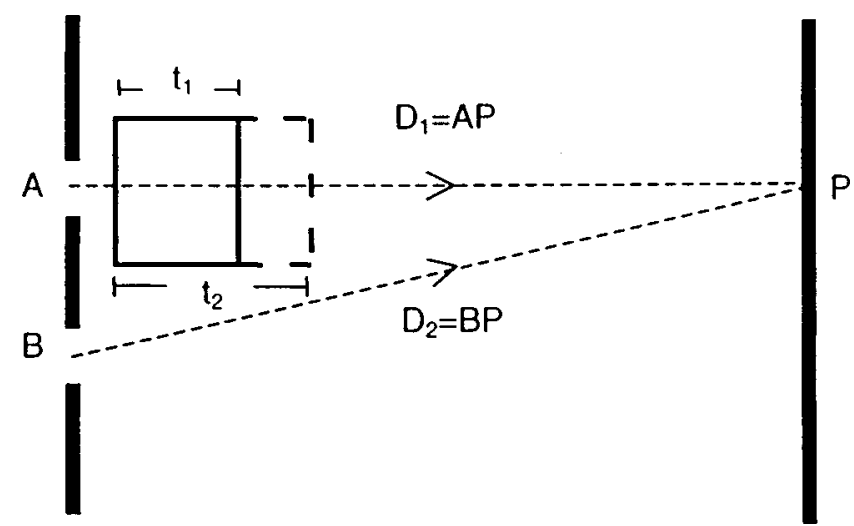

FIG. 1. Schematic of the double-slit arrangement for transmission fringes. The film thickness is shown highly exaggerated.

Transmission fringes: In this arrangement, a laser is incident on a double slit with a film placed in front of one of the slits (see Fig. 1). When the beams originating at $A$ and $B$ meet, they produce a pattern of interference fringes on a screen. The optical path difference (OPD) between the two beams for a point $P$ on the screen is given by

$$
\begin{aligned}
(\mathrm{OPD})_{1}= & {\left[\left(D_{1}-t_{1}\right)+n_{1} t_{1}\right] } \\
& -D_{2} \text { (before temperature change), }
\end{aligned}
$$

where $D_{1}$ is the geometrical distance from $A$ to $P, D_{2}$ is the distance from $B$ to $P, n_{1}$ is the initial refractive index of the film, and $t_{1}$ is the initial thickness. There will be a bright or dark fringe at $P$ depending on whether this optical path difference is an integral or a half-integral multiple of $\lambda$, the wavelength of light. When the film is heated, the optical path length of the beam originating at $A$ changes, causing a shift in the fringe pattern. If the refractive index and thickness of the film after heating are $n_{2}$ and $t_{2}$,

$$
\begin{aligned}
(\mathrm{OPD})_{2}= & {\left[\left(D_{1}-t_{2}\right)+n_{2} t_{2}\right] } \\
& -D_{2}(\text { after temperature change })
\end{aligned}
$$

From Eqs. (1) and (2),

$$
\Delta(\mathrm{OPD})=(\mathrm{OPD})_{2}-(\mathrm{OPD})_{1}=\Delta(n t)-\Delta(t)=\left(\Delta m_{T}\right) \lambda,
$$

where $\Delta(n t)=n_{2} t_{2}-n_{1} t_{1}$ and $\Delta(t)=t_{2}-t_{1} \cdot \Delta m_{T}$ is the change in the order of the transmission fringe at the point $P$, due to the shift in the fringe pattern on heating the film. For instance, if during heating $\Delta$ (OPD) was equal to $\lambda$, then one entire fringe would pass by a photodetector placed at point $P$.

Reflection fringes: For our second method, we use the interference of the beams reflected off of the front and back faces of the film, as shown in Fig. 2. For a given angle of incidence $i$ of the beam on the film, the optical path length is dependent only on the refractive index and the thickness. It can be easily derived that

$(\mathrm{OPD})_{1}=2 t_{1}\left[n_{1}^{2}-\sin ^{2}(i)\right]^{1 / 2}$

(before temperature change),

$(\mathrm{OPD})_{2}=2 t_{2}\left[n_{2}^{2}-\sin ^{2}(i)\right]^{1 / 2}$

(after temperature change).

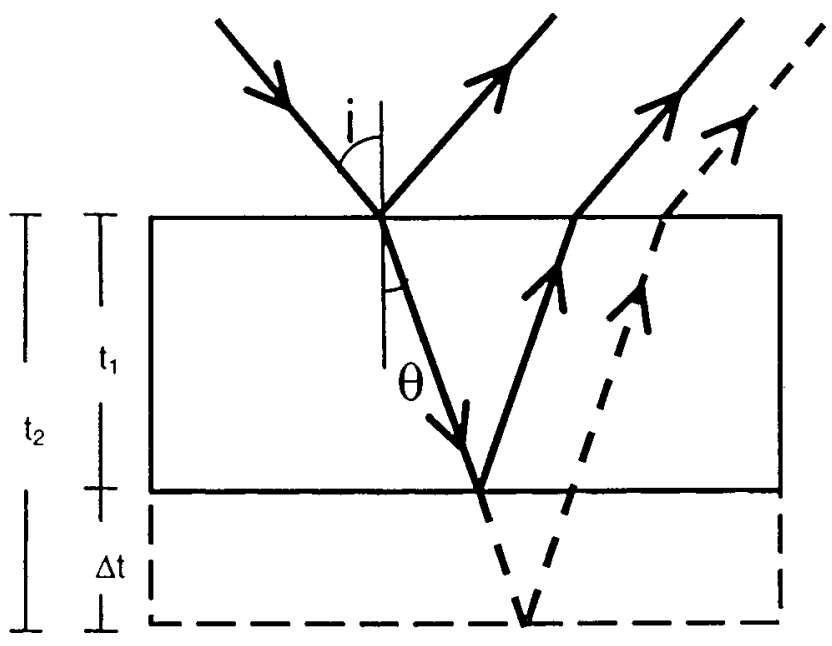

FIG. 2. Schematic representation of the reflection fringes. The dashed lines represent the situation for the expanded (heated) film.

From Eqs. (4) and (5), for small $i$,

$$
\Delta(\mathrm{OPD})=(\mathrm{OPD})_{2}-(\mathrm{OPD})_{1} \cong 2 \Delta(n t)=\left(\Delta m_{R}\right) \lambda .
$$

It should be noted from Eqs. (3) and (6) that the transmission fringes are sensitive to $\Delta(n t)-\Delta(t)$, while the reflection fringes, for low angles of incidence, are sensitive to $\Delta(n t)$. Further, if $n_{1}, t_{1}$ are the refractive index and thickness of the film at temperature $T_{1}$, and $n_{2}, t_{2}$ their values at temperature $T_{2}$, then,

$$
\begin{aligned}
& n_{2}=n_{1}+n_{1} \alpha_{n}\left(T_{2}-T_{1}\right), \\
& t_{2}=t_{1}+t_{1} \alpha_{t}\left(T_{2}-T_{1}\right),
\end{aligned}
$$

where $\alpha_{n}$ and $\alpha_{t}$ are the temperature coefficients of the thickness change and refractive index change, respectively. $\alpha_{t}$ is positive, whereas $\alpha_{n}$ can be either positive or negative depending on the material and the temperatures involved. The working equations can be derived by combining Eqs. (3), (6), (7), and (8), and putting $\Delta T=T_{2}-T_{1}$,

$$
\begin{aligned}
& {\left[\left(n_{1}-1\right) \alpha_{t}+n_{1} \alpha_{n}\right] t_{1} \Delta T=\left(\Delta m_{T}\right) \lambda,} \\
& {\left[2 n_{1}\left(\alpha_{n}+\alpha_{t}\right)\right] t_{1} \Delta T=\left(\Delta m_{R}\right) \lambda .}
\end{aligned}
$$

Our experimental design incorporates the two ideas discussed above. A He-Ne laser is directed on a beam splitter, as shown in Fig. 3. One of the two beams from the beam splitter passes through the film while the other half is diverted to a mirror a small distance away (see Figs. 3 and 4). The mirror directs the beam into the path of the transmitted beam so that the two will eventually meet and interfere at the first magnifying lens. One of the two interfering beams passes through the film. Placing the mirror close to the beam splitter helps to increase the fringe spacing. The film is surrounded by a brass plate on either side. The brass plates have windows and the film is mounted part of the way into the window, as shown in Fig. 4. One of the interfering beams passes through the part of the window interposed by the film, while the second interfering beam passes clear of the film through the same window. The brass plates are wrapped with heater bands and insulated with fiberglass insulation. This is part of the temperature control system that is used to set and 


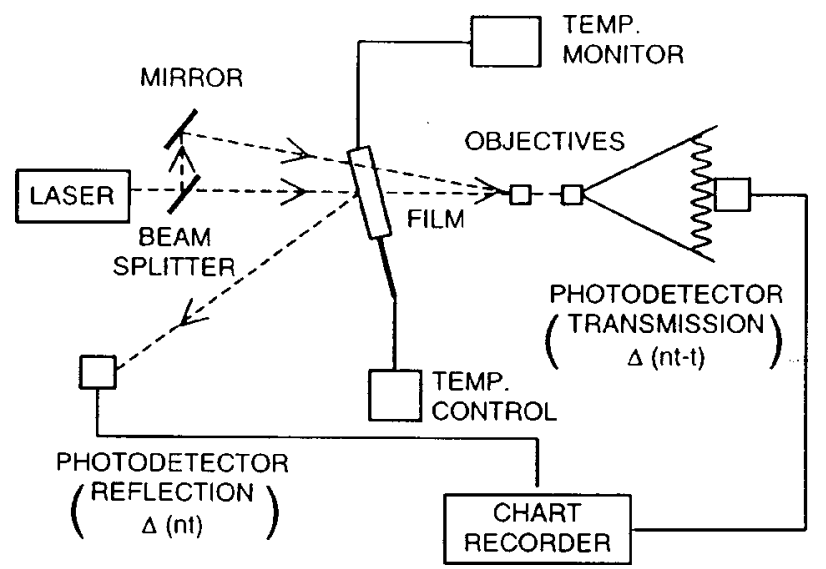

FIG. 3. Schematic diagram of the experimental setup. The angle of incidence of the laser beam on the film is shown exaggerated for clarity.

control the temperature of the film. A thermistor attached to the film measures its temperature directly. Microscope objectives are used to magnify the interference pattern. A photodiode is used as a detector. Very sharp, high contrast interference fringes can be seen on a screen placed in the plane of the photodetector. The reduction in intensity of the beam through the film is small and does not affect the sharp visibility of the fringes. If it becomes a problem, a neutral density filter can be introduced in the path of the other beam to compensate for this and restore sufficient contrast needed for the measurements. On heating the film, the fringe pattern shifts and the light incident on the photodiode alternates between bright and dark, producing waves as the output on the chart recorder. When the recorder moves from one peak to the next, the optical path difference between the two beams has changed by $632.8 \mathrm{~nm}$, the wavelength of the light from the $\mathrm{He}-\mathrm{Ne}$ laser. The film is oriented at a low angle of incidence, so that the beam that is reflected off of it will reach the detection setup, as shown in Fig. 3. After passing through a magnifying lens, the reflected pattern is detected by a photodetector and observed on a chart recorder. The fringe patterns can also be monitored with a video camera. After recording the progress of the pattern as it moves across the screen, it is possible to fast forward the tape and actually count the fringes that pass. From the traces on the chart recorder, the fringe shifts are obtained to within an eighth of a fringe. The fringe pattern is very sensitive to both air currents and mechanical vibrations. We used a floated optical table and carefully shielded the beam path from air currents.

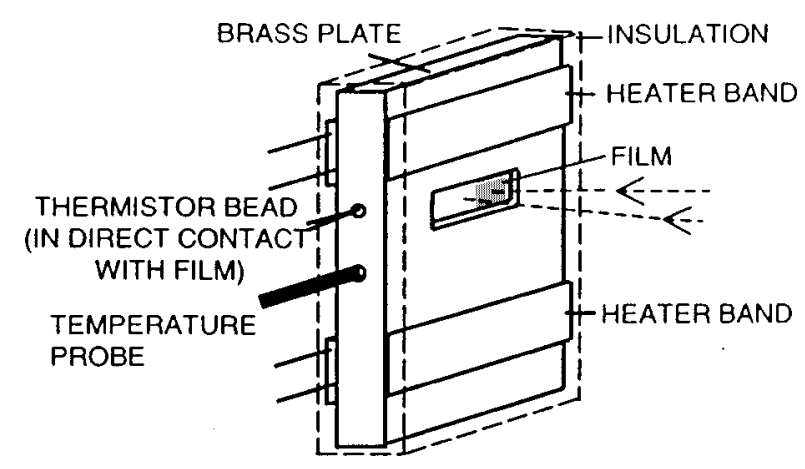

FIG. 4. Details of the film encasement.
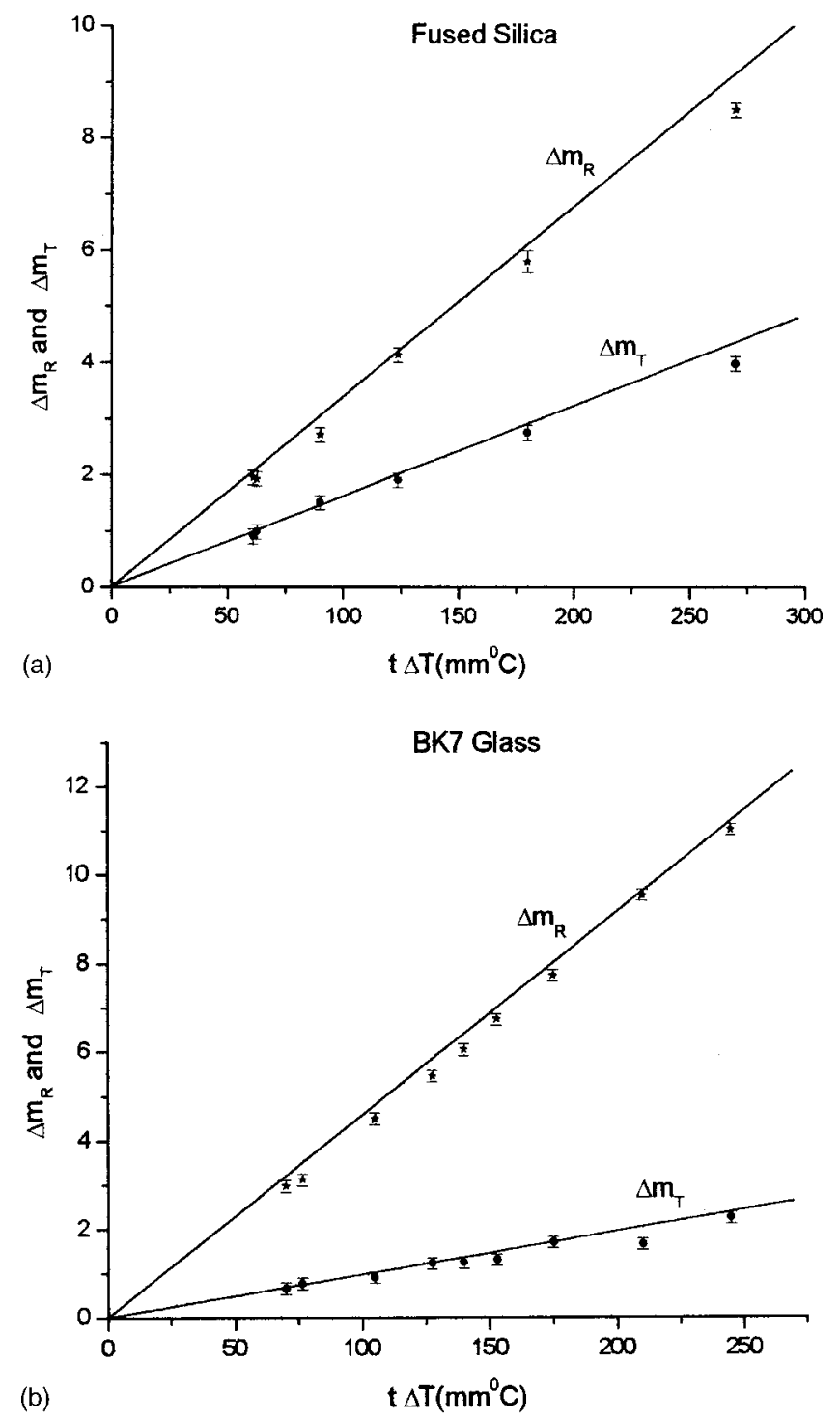

FIG. 5. (a) Fused silica: graph of the measured $\Delta m_{R}$ and $\Delta m_{T}$ against the product of sample thickness and temperature change. The error bars represent an eighth of a fringe. The expected line, calculated from the known values of $\alpha_{n}$ and $\alpha_{t}$ for fused silica, is also shown for comparison with the data. (b) BK7 glass: graph of the measured $\Delta m_{R}$ and $\Delta m_{T}$ against the product of sample thickness and temperature change. The error bars represent an eighth of a fringe. The expected line, calculated from the known values of $\alpha_{n}$ and $\alpha_{t}$ for BK7 glass, is also shown for comparison with the data.

Differential heating of the air in the two interfering beam paths would lead to a shift in the fringe pattern on heating, even without the film. We were able to eliminate the effect of the differentially heated air as follows: instead of maneuvering the reflected beam entirely around the film encasement, we moved the film so that it covered only half of the window in the brass plate and then steered the reflected beam through the other half. Thus, by bringing the beams closer together, we were able to reduce the drift that occurred when heating without a film to less than half a fringe over a change in temperature of $75^{\circ} \mathrm{C}$.

We used two different types of glasses: Fused silica and BK7 glass. The fused silica pedigree glass samples were obtained from Corning Inc. (Corning code 7940). BK7 glass (International code 517642) samples were obtained from 
Schott Glass. The two types of glasses differ greatly in the values of their refractive indices and thermal expansion coefficients, as seen below. Fused silica has a very low thermal expansion coefficient and is used in optical components where this feature is an asset.

The values of $\alpha_{n}$ and $\alpha_{t}$ for the two glasses as supplied by the manufacturers are listed below.

Fused silica: $\alpha_{n}=6.9 \times 10^{-6} /{ }^{\circ} \mathrm{C}$

and

$$
\begin{aligned}
& \alpha_{t}=0.5 \times 10^{-6} /{ }^{\circ} \mathrm{C}, \\
& \text { BK7: } \alpha_{n}=1.25 \times 10^{-6} /{ }^{\circ} \mathrm{C}
\end{aligned}
$$

and

$$
\alpha_{t}=8.3 \times 10^{-6} /{ }^{\circ} \mathrm{C} .
$$

Figures 5(a) and 5(b) show the measured shifts in transmission and reflection fringes plotted against the product of the thickness and the temperature $(t \Delta T)$ for each of the two glasses. As seen, the data fit very well with the calculated fringe shifts in each case. Values of $\alpha_{n}$ and $\alpha_{t}$ calculated from the measurements using a linear fit to the data and Eqs. (9) and (10) are as below:

$$
\begin{gathered}
\text { Fused silica: } \alpha_{n}=(6.4 \pm 0.2) \times 10^{-6} /{ }^{\circ} \mathrm{C} \text { and } \\
\alpha_{t}=(0.64 \pm 0.10) \times 10^{-6} /{ }^{\circ} \mathrm{C}, \\
\text { BK7: } \alpha_{n}=(0.94 \pm 0.1) \times 10^{-6} /{ }^{\circ} \mathrm{C} \text { and } \\
\alpha_{t}=(8.3 \pm 0.2) \times 10^{-6} /{ }^{\circ} \mathrm{C} .
\end{gathered}
$$

The data corresponding to the largest value of $t \Delta T$ has been omitted in the fits for the above calculations. It is important to note from Eq. (9) that the transmission fringes are relatively more affected by refractive index changes than thickness changes. Hence, for materials with a much larger $\alpha_{n}$ compared to $\alpha_{t}$, the fringe shifts are dominated by the index changes and, hence, the resulting error bar on the measured value of $\alpha_{t}$ becomes larger. This would limit the usefulness of this technique in such cases. The good agreement obtained in the case of the samples that we have measured confirms the feasibility and use of this technique. The key parameter that would determine the applicability of the method to a sample comes from the fact that the absolute fringe shifts produced by the film for changes in temperature of a few tens of degrees should be large enough compared to the precision of the fringe-shift measurements. Previous measurements of transmission fringe shifts using Kapton ${ }^{19}$ show that it is possible to stack films to increase the absolute fringe shifts in the case of very thin films or films with very low thermal coefficients. The fringe shifts obtained in the transmission for a stack of three films was found to be three times that for a single film, thus providing a way to increase absolute fringe shifts and slightly extending the applicability of the method to thinner samples. ${ }^{19}$

In conclusion, a successful solution to the problem of decoupling of the two coefficients $\alpha_{n}$ and $\alpha_{t}$ using optical interferometric methods has been demonstrated. The coefficients can be calculated directly from the data.

The authors would like to thank Christine J. Ofcarcik for the preliminary work on this experiment. The authors further acknowledge the support from Smith College and the NSF Materials Research Science and Engineering Center at the University of Massachusetts under cooperative agreement No. DMR9400488.

${ }^{1}$ O. S. Heavens, Optical Properties of Thin Solid Films (Dover, New York, 1965).

${ }^{2}$ K. L. Saenger and H. M. Tong, New Characterization Techniques in Thin Polymer Films (Wiley, New York, 1990).

${ }^{3}$ P. Nachman, Am. J. Phys. 61, 564 (1993).

${ }^{4}$ T. Ohyama and Y. H. Mori, Rev. Sci. Instrum. 58, 1860 (1987).

${ }^{5}$ T. Ohyama, K. Endoh, A. Mikami, and Y. H. Mori, Rev. Sci. Instrum. 59, 2018 (1988)

${ }^{6}$ C. J. Parker and W. A. Popov, Appl. Opt. 10, 2137 (1971).

${ }^{7}$ H. G. Tompkins, User's Guide to Ellipsometry (Academic, Boston, MA, 1993).

${ }^{8}$ H. G. Tompkins and W. A. McGahan, Spectroscopy and Reflectometry: A User's Guide (Wiley, New York, 1999).

${ }^{9}$ R. M. A. Azzam and N. M. Bashara, Ellipsometry and Polarized Light (North-Holland, Amsterdam, 1979).

${ }^{10}$ J. Cao, M. Yanagihara, and M. Yamamoto, Appl. Opt. 33, 2013 (1994).

${ }^{11}$ Z. Gu, P. Liang, and X. Liu, Meas. Sci. Technol. 11, N56 (2000).

${ }^{12}$ K. Etoh, Appl. Opt. 34, 159 (1995).

${ }^{13}$ M. Nenkov and T. Pencheva, J. Opt. Soc. Am. 15, 1852 (1998).

${ }^{14}$ I. Chambouleyron, J. M. Martinez, and A. C. Moretti, Appl. Opt. 36, 8238 (1997).

${ }^{15}$ C. Wang, X. Shao, and J. Mao, Meas. Sci. Technol. 8, 911 (1997).

${ }^{16}$ P. Meredith, G. S. Buller, and A. C. Walker, Appl. Opt. 32, 5619 (1993).

${ }^{17}$ J. Reyes, E. Marquez, and J. B. Ramirez-Malo, J. Mater. Sci. 30, 4133 (1995).

${ }^{18}$ R. P. Shukla, A. Chowdhury, and P. D. Gupta, Opt. Eng. (Bellingham) 33, 1881 (1994).

${ }^{19}$ C. J. Ofcarcik, Honors thesis, Smith College (1993). 